\title{
Overview of Wound Features and Treatment in Wenchuan Earthquake Victims
}

\author{
Yuanzhang Yao*, Lianyang Zhang, Xiaobin Cheng, Yue Shen, Yong He \\ The Center of War Wound and Trauma, PLA, Research Institute of Surgery, Daping Hospital, The Third Military \\ Medical University, Chongqing, China \\ Email: ${ }^{*}$ Yaoyz007@163.com
}

Received 20 March 2014; revised 19 April 2014; accepted 9 May 2014

Copyright (C) 2014 by authors and Scientific Research Publishing Inc.

This work is licensed under the Creative Commons Attribution International License (CC BY).

http://creativecommons.org/licenses/by/4.0/

(c) $\underset{\mathrm{EY}}{\mathrm{i}}$ Open Access

\section{Abstract}

Objective: "5.12" Wenchuan Earthquake killed 70,000 people and left more than 90,000 people injured seriously. This study was to analyze the wound features and early treatments in Wenchuan earthquake victims and investigate treatment strategies in major disasters, to provide a reference to future disaster rescue. Methods: Total of 1420 earthquake patients who were admitted to Deyang People's Hospital, Sichuan Province from May 12-30, 2008 were enrolled in the study. The wound features and early treatment were retrospectively analyzed. Results: Among 1420 patients, there were total 1821 injuries. In which, there were 1089 cases with single site injury (76.69\%) and 331 cases with multiple injury (23.31\%); injuries of limb, body surface and soft tissue were more than that of other sites; injury of single site was larger than that of multi-site $(P<$ 0.01); most of the earthquake patients were admitted to hospital within 2 days after earthquake $(68.80 \%)$, whose number was more than those at other times $(P<0.01)$; the number of the earthquake patients in 19 - 45 age group was more than that of other groups $(P<0.01)$; most of patients were treated within 12 hours after injury $(P<0.01)$. Conclusion: In disaster rescue, rescue speed is the key point. And the "golden time" and emphases of rescue should be within 2 days after earthquake; the principle of damage control surgery should be followed in the rescue. Enhancing mutual and self help practice and first-aid training at usual times may save more lives.

\section{Keywords}

Wenchuan, Earthquake, Disaster Medicine, Injury, Rescue, First Aid, Strategy

\footnotetext{
${ }^{*}$ Corresponding author.
} 


\section{Introduction}

A magnitude 8.0 earthquake struck China's southwestern Sichuan province at 14:28 May 12, 2008. This was the most costly and wide spread natural disaster since the foundation of China. By the end of 12:00 July 6, 2008, the earthquake had killed 69,196 people, caused 18,379 missing and left 374,176 injured. A total of 96,419 people were hospitalized due to injury. Meanwhile, a number of cities and villages were ruined and a majority of hospitals and medical equipments were destroyed. Fifteen hours after the earthquake, the first medical team from the Third Military Medical University (TMMU) arrived at Deyang, Sichuan province which was 50 kilometers from the epicenter. The team which was composed of expert group and treatment group joined in the rescue and treatment of the wounded in earthquake and was one of the first medical teams outside the province to arrive at Deyang. During nearly a month of rescue, the medical team, combined with Deyang People' Hospital (DPH), had treated a vast number of patients injured in the earthquake.

This study was to investigate the early treatment strategies in major disasters through an analysis of wound features, the wounded flow and early treatment of 1420 patients admitted to DPH in the earthquake on May 12.

\section{Materials and Methods}

We retrospectively analyzed the original medical records of 1420 patients admitted to the medical team and DPH from May 12-30. The injury time and place, injury mechanism, age, gender, occupation, injury site, injury type, diagnosis, treatment method and prognosis of the wounded were recorded.

\subsection{Composition and Development of the Medical Team}

The medical team was stationed in DPH which was 50 kilometers from the epicenter. Being equipped with the common emergency drugs and instruments as well as a field operation vehicle, the medical team could carry out two large or moderate operations at the same time. The team contained 35 members. In which, 3 were administrators, 27 were experts and doctors who specialized in trauma, emergency, orthopedics, cardiothoracic surgery, cerebral surgery, anesthesia, intensive care and laboratory examination and 5 were logistics support personnel. The medical team was a standing force for the TMMU to deal with health emergencies, which had experienced multi-year military medical affair training and frequently involved in many medical rescues in peacetime and wartime.

Medical equipments and houses of DPH were ruined in the earthquake, but no casualties occurred. In order to change the disorder of rescue and clearly define the responsibilities of DPH's staff within the first 24 hours after the earthquake, the medical team, after arriving at Deyang at 5:00 a.m. May 13, immediately coordinated with DPH and local health administrative department. The implementation of rescue effort would rely mainly on the military medical team while DPH subsidiary by combining with local situation and medical treatment platform of DPH.

\subsection{Division of the Areas for Rescue}

The areas for rescue were divided according to the geographic environment of DPH. The size of areas and staff disposition was determined by the flow of the wounded. It was divided into: 1) triage area: located in front of the gate and in the hall of emergency department, responsible for patient reception, triage, categorization, rapid injury assessment and emergency treatment; 2) examination area: temporally established in the hall of emergency department, $20 \mathrm{~m}$ from the triage area, responsible for rapid examination of B-ultrasound, Electrocardiogram (ECG) and X-ray, and Tetanus Antitoxin (TAT) injection; 3) operation area: $50 \mathrm{~m}$ from the triage area, located in the tent behind triage area, and 3 - 4 debridements could be performed at the same time and two operation tables were set in the field operation vehicle where two large or moderate operations could be performed simultaneously; 4) observation area: 100 - $300 \mathrm{~m}$ from the triage area, responsible for the observation, hospitalization and ICU care of the treated patients. According to the different responsibilities and missions, 4 military doctors, 5 local doctors, 6 nurses and 8 volunteers allocated to each area should be ensured.

\subsection{Triage}

Four military doctors from the medical team who had been engaged in military medical affair training for a long 
time and experienced in injury treatment led 2 - 3 doctors, 4 nurses from the DPH and several volunteers to form a team. They categorized and measured the patients who were left stranded at the emergency area or was currently hospitalized, rapidly assessed and diagnosed injury conditions of the patients, and then established treatment measures and determined priority of treatment. Based on the priorities, an information card with different colors was attached to the chest or the arm of the wounded. Black represented dead patients; red represented most seriously wounded patients (such as open injury accompanied by hemorrhage, shock, severe traumatic brain injury, thoraco-abdominal injury, unstable vital signs and patients who needed an emergency operation or care in ICU); yellow represented seriously wounded patients (such as a severe closed fracture which was not necessary for an operation immediately and the patients who had open injury of soft tissue that needed debridement and stable vital signs); green represented lightly wounded patients who transiently did not need operation; and blue represented co-infected wounded patients. The card presented basic information of the patients, such as name, gender, age, injury time, injury site, injury mechanism, primary diagnosis, treatment after injury, examination and treatment proposal, and whereabouts, which would provide a reference for the subsequent treatment. After the triage was finished, the patients should be rapidly shunted.

\subsection{Record of the Injury Site}

Human body was divided into nine anatomical regions according to AIS-2005 version, namely, head, face and cervical region, chest, abdomen, spine and spine cord, pelvis, four limbs (upper limbs and lower limbs) and body surface. The incidence of injury of each site was compared.

\subsection{Record of the Wound Features}

Curve of the wounded flow was drawn according to treatment conditions in recent 20 days; the wound features of the patients were analyzed. The patients were divided into the following groups by age: preschool children group ( $<7$ yrs), student group ( 7 - 19 yrs), middle-aged and young group (20 - 45 yrs), middle-aged and elderly group (46 - 65 yrs) and elderly group (>65 yrs). The number of the wounded in each age group was compared. The injuries were divided into multiple injuries, crush injury, incised injury, blunt injury, combined injuries; the injuries were divided into skin and soft tissue injury (contusion, abrasion and lacerated injury), limbs fracture and mixed injury, according to injury types.

\subsection{Treatment Methods}

Treatment methods were categorized according to the time of initial treatment and treatment method for the patients after injury: 1) field first aid: the treatment was performed within 10 minutes after injury, including ventilation, hemostasis, bandaging, fixation and transportation; 2) emergency treatment: treatment was performed within 3 hours after injury, including thoracic close drainage and bandaging for the prolapsed viscera, besides the content in field first aid; 3) early treatment; treatment was performed within 6 hours after injury, including debridement, amputation for the damaged limb, incision of trachea, thoracic close drainage, thoracic surgery, laparotomy, antishock and anti-infection therapy; 4) special treatment: treatment was performed within 12 hours after injury, including established amputation, enucleation of eyeball, vascular repair, brain debridement, repairing operation for the organs in thoracic and abdominal cavity, dialysis for the crush syndrome patients, systemic anti-shock and anti-infection therapy and open reduction and internal fixation for the closed fracture; 5) later stage treatment: treatment was performed within 12 hours after injury, including field first aid, emergent treatment, early treatment and special treatment.

\subsection{Statistical Analysis}

The statistical analysis was conducted by SPSS10.0. The chi-square test was used to compare the rates and t-test was used to compare multiple rate. $P<0.01$ was considered to be statistically significant.

\section{Results}

From May 12-30, 2008, a total of 1420 patients were hospitalized, accounting for $2.57 \%$ of all the wounded from Deyang district $(55,255)$ and $5.11 \%$ of all the hospitalized patients $(27,800)$. In 1420 patients, 652 were 
males and 768 were females; and their age range was 0.2 - 102 years and mean age was $43.70 \pm 12.16$ years. Injury mechanism was: those injured by the collapsed house (95.07\%), by fall during escape (3.87\%) and others, such as injured in the rescue (1.06\%). Geographical distribution was: 94 cases (6.62\%) in Deyang city, 1182 cases (83.24\%) in Mianzhu city, 103 cases (7.25\%) in Shifang city and 41 cases (2.89\%) in other districts.

\subsection{The Wounded Patient Flow}

Most wounded patients, total 977 cases, were admitted to the hospital within 2 days after the earthquake, accounting for $68.80 \%$ of the total patients. The wounded patient flow was shown in Figure 1 . The number of admitted victims within 2 days after the earthquake was significantly larger than that at other times $(P<0.01$, chisquare test).

\subsection{The Age Range of the Wounded}

Injury condition of different age groups was listed in Table 1. It showed that the patients in 19 - 45 age group were most, followed by those in 46 - 65 age group and those in preschool children age group (less than 7 years) were least. There were statistically significant differences between the age groups $(P<0.01$, t-test).

\subsection{Injury Site}

The incidence of injury at each site was shown in Table 2. It showed that injuries of limb, body surface and soft tissue were more than the injuries of other sites; face and cervical injuries were least. There was significant difference in the incidence of injuries of different sites $(P<0.01$, t-test). There were 1821 injuries in 1420 patients. The single site injury reached $76.69 \%(n=1089)$ and multi-site injury accounted for $23 \%(n=331)$; the former was significantly more than the latter $(P<0.01$, chi-square test).

\subsection{The Wound Features}

The open injuries and closed injuries accounted for $61.55 \%(n=874)$, the simple open injury for $21.90 \%(n=$ $311)$ and closed injury for $16.55 \%(n=235)$. There was statistically significant difference between these groups $(P<0.01$, t-test); the multiple injuries and thoraco-abdominal injuries mainly presented within 24 hours after the earthquake, accounting for $22.59 \%$ of the treated patients admitted in the same period; patients with crush injury

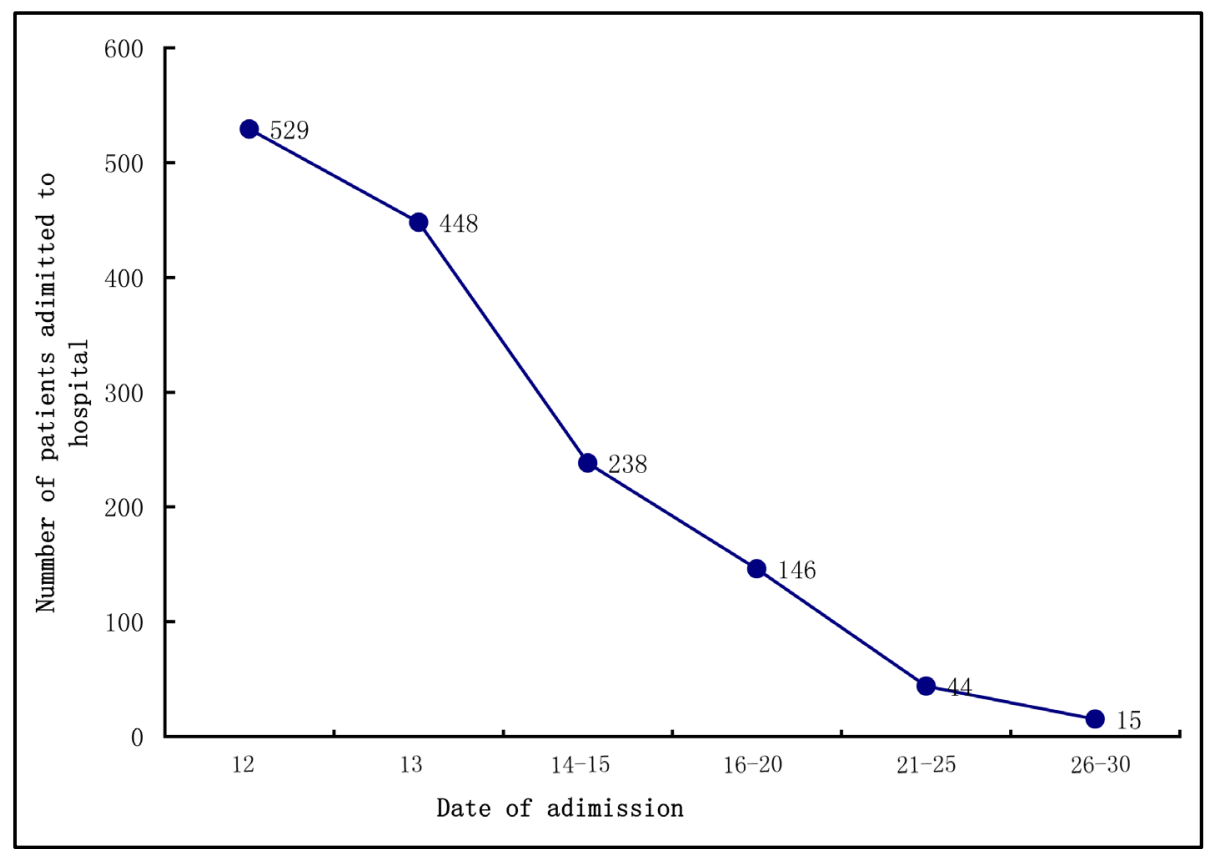

Figure 1. Wounded flow in DPH from May 12 to 30. 
Table 1. Number of the hospitalized patients in each age group.

\begin{tabular}{ccc}
\hline Age group & No. of hospitalized patients (n) & Constituent ratio (\%) \\
\hline$<$ & 43 & $3.03 \%$ \\
$\mathbf{7}-\mathbf{1 8}$ & 215 & $15.14 \%$ \\
$\mathbf{1 9}-\mathbf{4 5}$ & 544 & $38.31 \%$ \\
$\mathbf{4 6}-\mathbf{6 5}$ & 331 & $23.31 \%$ \\
$>\mathbf{6 5}$ & 287 & $20.21 \%$ \\
Total & $\mathbf{1 4 2 0}$ & $\mathbf{1 0 0}$ \\
\hline
\end{tabular}

Table 2. Anatomical injury site of 1420 patients.

\begin{tabular}{ccc}
\hline Injury site & No. of the wounded (n) & Constituent ratio (\%) \\
\hline Head & 157 & $11.06 \%$ \\
Face and cervical site & 32 & $2.25 \%$ \\
Chest & 142 & $10.00 \%$ \\
Abdomen & 42 & $2.96 \%$ \\
Pelvis & 74 & $5.21 \%$ \\
Spine and spine cord & 123 & $8.66 \%$ \\
Limbs & 746 & $52.54 \%$ \\
Body surface and soft tissue & 505 & $35.56 \%$ \\
Total & $\mathbf{1 8 2 1}$ & $\mathbf{1 2 8 . 2 4 \%}$ \\
\hline
\end{tabular}

*Note: 331 patients had injuries of more than 2 anatomical sites.

and crush syndrome were mainly hospitalized 48 hours after the earthquake, accounting for $3.80 \%(n=54)$, and 46 cases were crush in lower limbs, 18 cases in upper limbs. Injury types were: limbs fracture $52.54 \%$ and skin and soft tissue injury (contusion, abrasion and lacerated injury) 35.56\%, which were more than other types of injury $(P<0.01$, t-test). The injury severity was: slight injury (AIS value: $\leq 2) 62.89 \%(n=892)$, severe injury (AIS value: $3-4) 32.75 \%(n=465)$, most severe injury (AIS value: $>4) 4.43 \%(n=63)$; there was statistically significant difference between these groups $(P<0.01$, t-test).

\subsection{Treatment Contents}

Treatment provided by the medical team and DPH to the wounded covered emergency treatment, early treatment and special treatment. The wounded patients who received treatment (including field first aid) before hospitalization and were transferred to DPH accounted for $25.49 \%(n=362)$; those who had not undergone field treatment and were transferred to DPH accounted for $74.51 \%(n=1058)$. The treatment conditions in the hospital were shown in Table 3. It showed that a majority of victims were treated over 12 hours after injury. There was statistically significant difference between the groups $(P<0.01$, t-test).

\subsection{Prognosis}

In 1420 patients, 1372 survived, accounting for $96.62 \%$ of the total patients; 48 died (3.38\%); 42 died before hospitalization or after emergency treatment, who appeared within 24 hours after injury (cause of death was unknown), and 6 patients died after hospitalization (3 cases for severe traumatic brain injury, 3 cases for multiple organ failure). 
Table 3. The treatment conditions of directly transferred patients.

\begin{tabular}{ccc}
\hline Time after injury (h) & Treatment contents & No. of cases (\%) \\
\hline$<3$ & Emergency treatment & $47(4.44 \%)$ \\
$3-6$ & Early treatment & $108(10.21 \%)$ \\
$7-12$ & Special treatment & $384(36.29 \%)$ \\
$>12$ & Later stage treatment & $504(47.64 \%)$ \\
Total & & $1043(98.58 \%)$ \\
\hline
\end{tabular}

*Note: Fifteen patients were not treated or without medical records.

\section{Discussion}

According to the report, more than 50,000 earthquakes occur annually all over the world. However, most of them have slight impact and only a few of them lead to casualties and property loss. In the past two decades, nearly 3 million people died in earthquake 1 Wenchuan earthquake was another serious natural disaster in China following Tangshan earthquake in 1976, which caused an unprecedented devastation, impact and property loss.

This study was to evaluate the epidemiological data of victims in " 5.12 " earthquake treated by the first medical team of the TMMU and DPH, to discuss the wound features and treatment required by the wounded and the mode of military medical team to participate in disaster medical rescue in peacetime, in order to provide a reference for the disaster medical rescue in developing countries.

Injury treatment at usual times emphasize on timeliness and integrity. Speed is the key factor for successful treatment. The concept such as "platinum 10 minutes" and "golden 1 hour" has been widely accepted by people. The feature of the disaster rescue in Wenchuan earthquake was that the urban victims could receive treatment within 3 - 6 hours after injury. However, a majority of patients were distributed in rural areas. When information, road and medical source were unavailable, the timeliness of treatment could not be guaranteed. Most victims could not receive timely treatment so that a number of severely injured patients lost the chance for treatment. Therefore, the philosophy of injury treatment at usual times is facing great challenge. This was also the reason that the most seriously injured patients were few within 2 - 3 days after the earthquake (the patients with thoraco-abdominal injury were few, and most of them had been dead before hospitalization) and the patients with non-fatal injury were more.

From the patients wounded and their treatment conditions in recent 20 days, the patients were most within 2 days after the earthquake, but more than 95\% patients were treated within 1 hour after injury. Nearly 500 victims were gathered within 3 - 12 hours after injury, and most of them needed surgical procedure and emergent treatment. Therefore, we considered that the treatment of the wounded in this disaster was different from that at usual times and the golden time and emphases of the treatment should fall into the first 2 days after the earthquake. At this time, the medical rescue team should arrive at the disaster area within 24 hours after the earthquake and rapidly start rescue in order to exert a positive and effective role. In November 2003, an earthquake broke out in Bam area, Iran. The Iran army bears the medical and rescue mission in the earthquake. Iran army, as the first rescue force, implemented seeking and rescue activities within 1 hour after the earthquake. The army set up two field hospitals which acted as the First Field Hospital at the first time, showing a strong ability in the early rescue action and playing a special role in the seeking and rescue [1]. In a summary of the medical rescue for Thailand tsunami in 2004, the importance of reconstructing the health system of the disaster area within 24 hours and intensifying field medicine aid were also stressed [2]. The earthquake rescue experience in Pakistan in August 2005 indicated that the military field surgical hospital could rapidly start humanitarian relief mission. Although the national disaster prevention division and WHO was suspicious of the use of military field surgical hospital, its effects on the early stage treatment had been affirmed. Because several weeks after the earthquake, the patients that needed operation would decrease rapidly and those needed fundamental medical service would increase gradually, even up to $90 \%$, to expand the fundamental medical unit at the later stage of rescue should be stressed [3].

This study showed that the treatment of most of the wounded was within 72 hours after the earthquake. In this period, the equipments and houses of DPH were destroyed and the medical personnel were in panic, which 
could not meet the needs of the treatment of a vast number of patients. However, the first medical team of the TMMU was in good preparation and they carried enough drugs and good devices with them. Food, water and daily-necessities were self-sufficient, which, under the condition of no logistical support, could meet the needs of the patient treatment for 3 days after the earthquake. The medical team, cooperated with DPH, overcame the bad conditions of equipments and tired staff and worked around the clock, giving top priority to rescue the life of the victims. Based on the local medical institute, this kind of rescue pattern that medical team acted as an expert group and treatment group, closely worked with the large-scale hospital in the disaster area, could both carry out the emergent treatment of first-line wounded patient and organize the patients to move in or move out of the hospital. At the same time, the major medical infrastructures were reconstructed in the disaster area to control the trauma, disease and plague as much as possible through forming a local medical hub. This kind of rescue mode implemented an important disaster rescue method in which the local and the military force were combined together. Though the performance of early rescue was remarkable, the subsequent supplement of staff, device and items should not be neglected, which had been reported in the previous disaster rescues [4]-[6]. In the medical rescue of Wenchuan Earthquake, many kinds of medical rescue modes were adopted. We considered that the rescue action should be carried out flexibly according to the mission and target of the rescue. In Duzce Earthquake in Turkey in 1999, Israel defense force organized a field hospital composed of multi-disciplinary and specialties, which played an important role in providing medical service to the inhabitants in disaster area. It was considered that the military field hospital could take the place of the local hospital [7]. In the rescue practices of the Mitch Hurricane in Honduras (November 1998) and Salvador Earthquake (January 2001), the international medical rescue mode was varied, from self-sufficient military medical team to the expert group or specialist, and they all exerted important roles [8].

An analysis of injury conditions among different age groups showed that the number of wounded patients in preschool children, middle and primary school students and elderly people groups were less than that in other groups. It didn't represent that few of the people in these age groups were injured, but reflected that the people in these age groups could not help for themselves, few of them escaped and most of them have been dead after being buried. While the wounded in middle-aged and young group and middle-aged and elderly group was over $60 \%$, who had a better ability to deal with the unexpected events and had stronger self-help and mutual-help capacity than the old, the weak and children. We suggested that 1) The self-help and mutual-help knowledge should be popularized to enhance the people's ability to deal with the sudden disaster and it was necessary to open a class in primary, middle school and university for exercising; 2) the field medical staff should master the knowledge and skills of emergency treatment to improve the treatment level for severe injuries and to set up a injury treatment force, which would had a better effect while dealing with the unexpected health event.

In the light of the features of anatomical injury site of the victims in earthquake (Table 2), which showed more open injury (21.90\%), more limbs fracture (52.54\%) and more soft tissue injury (35.56\%), the medical team should be made up of the doctors in trauma, emergency and general medicine; the first aid techniques such as ventilation, hemostasis, bandaging, fixation and transportation were still the emphases in the treatment. The first aid and emergent treatment techniques that were used most frequently were debridement, bandaging, fixation and transportation; two days after the earthquake, the correct diagnosis and treatment for the injury of thoraco-abdominal organs should be stressed to prevent missing diagnosis and misdiagnosis. The later stage treatment should mainly focus on the treatment of complications such as infection.

This study showed that about half of the victims presented limb injury (52.54\%), which were similar to the injury features that was reported in Japanese, Taiwanese and American earthquake in recent ten years [9]-[11]. $3.80 \%$ patients with crush injury presented crush syndrome, which mainly occurred in the patients who were hospitalized 48 hours later after the earthquake, which was close to the incidence of $2 \%$ - $5 \%$ reported by Sheng [12]. Acute renal failure was a reversible intermediate stage complication, which was usually related to the long time of bury, failure of timely rescue, increased environmental temperature and dehydration of the wounded [13].

As a result of a continuous influx of a large number of the wounded, in the first 24 hours after the earthquake, many patients waited for the emergent operation. When medical resources fail to exert function and to meet the usual treatment requirements (such as requirements of aseptic operation and selective operation), a decisive damage control operation will help to save more patients' lives. On the contrary, if a routine and time-consuming repair operation such as complicated blood vessel and nerve repair or resection of the combined organs was performed, more patients would lose the chance of treatment. This idea of damage control surgery was different 
from that at usual times. It was not to prevent the “dangerous triangle” [14] induced by low body temperature, coagulation disorders and metabolic acidosis, but to provide treatment to those victims who urgently needed operation as much as possible. At this time, a series of simple and effective measures such as debridement, hemostasis, ligation, external fixation, decisive amputation, rapid thoracic and abdominal cavity closure should be adopted for preliminary treatment so as to save lives as soon as possible. 24 - 48 hours later, according to the conditions of the wounded flow, a determinative treatment could be performed.

\section{Conclusion}

In conclusion, in the disaster rescue at usual times, rescue speed is the key factor. The golden time and emphases of the rescue should be within 2 days after the earthquake. In the earthquake injury, the open plus closed injury was most in number, and the limb and soft tissue injury was most common to see. The rescue should be carried out flexibly according to the mission and target of rescue. The damage control principle should be followed in the treatment. Strengthening mutual help and self help practice and first-aid training usually may save more lives.

\section{Acknowledgements}

The authors appreciate Dr. Jiang Wei, director of Emergency, Department and Deng Yanhua, head nurse of $\mathrm{DPH}$, Sichuan for their assistance in 5.12 earthquake rescue as well as data collection and arrangement in the later stage.

\section{References}

[1] Abolghasemi, H., Poorheidari, G., Mehrabi, A., et al. (2005) Iranian Military Forces in the Bam Earthquake. Military Medicine, 170, 859-861.

[2] Schwartz, D., Goldberg, A., Ashkenasi, I., et al. (2006) Prehospital Care of Tsunami Victims in Thailand: Description and Analysis. Prehospital and Disaster Medicine, 21, 204-210.

[3] Fernald, J.P. and Clawson, E.A. (2007) The Mobile Army Surgical Hospital Humanitarian Assistance Mission in Pakistan: The Primary Care Experience. Military Medicine, 172, 471-477.

[4] Bulut, M., Fedakar, R., Akkose, S., et al. (2005) Medical Experience of a University Hospital in Turkey after the 1999 Marmara Earthquake. Emergency Medicine Journal, 22, 494-498. http://dx.doi.org/10.1136/emj.2004.016295

[5] Chen, W.K., Cheng, Y.C., Ng, K.C., et al. (2001) Were There Enough Physicians in an Emergency Department in the Affected Area after a Major Earthquake? An Analysis of the Taiwan Chi-Chi Earthquake in 1999. Annals of Emergency Medicine, 38, 556-561. http://dx.doi.org/10.1067/mem.2001.119054

[6] Yoshimura, N., Nakayama, S., Nakagiri, K., et al. (1996) Profile of Chest Injuries Arising from the 1995 Southern Hyogo Prefecture Earthquake. Chest, 110, 759-761. http://dx.doi.org/10.1378/chest.110.3.759

[7] Bar Dayan, Y., Leiba, A., Beard, P., et al. (2005) A Multidisciplinary Field Hospital as a Substitute for Medical Hospital Care in the Aftermath of an Earthquake: The Experience of the Israeli Defense Forces Field Hospital in Duzce, Turkey, 1999. Prehospital and Disaster Medicine, 20, 103-106.

[8] Waisman, Y. (2003) Integration of Foreign and Local Medical Staff in a Disaster Area: The Honduras and El Salvador Experiences. European Journal of Emergency Medicine, 10, 124-129. http://dx.doi.org/10.1097/00063110-200306000-00011

[9] Collins, A.J. (1989) Kidney Dialysis Treatment for Victims of the Armenian Earthquake. The New England Journal of Medicine, 320, 1291-1292. http://dx.doi.org/10.1056/NEJM198905113201930

[10] Kuwagata, Y., Oda, J., Tanaka, H., et al. (1997) Analysis of 2702 Traumatized Patients in the 1995 Hanshin-Awaji Earthquake. Journal of Trauma, 43, 427-432. http://dx.doi.org/10.1097/00005373-199709000-00007

[11] Tanaka, H., Oda, J., Iwai, A., et al. (1999) Morbidity and Mortality of Hospitalized Patients after the 1995 HanshinAwaji Earthquake. American Journal of Emergency Medicine, 17, 186-191. http://dx.doi.org/10.1016/S0735-6757(99)90059-1

[12] Sheng, Z.Y. (1987) Medical Support in the Tangshan Earthquake: A Review of the Management of Mass Casualties and Certain Major Injuries. Journal of Trauma, 27, 1130-1155. http://dx.doi.org/10.1097/00005373-198710000-00007

[13] Mulvey, J.M., Awan, S.U., Qadri, A.A., et al. (2008) Profile of Injuries Arising from the 2005 Kashmir Earthquake: The First 72 h. Injury, 39, 554-560. http://dx.doi.org/10.1016/j.injury.2007.07.025

[14] Penninga, L., Penninga, E.I. and Svendsen, L.B. (2005) Damage Control Surgery in Multiply Traumatized Patients. Ugeskrift for Lager, 167, 3403-3407. 